OPEN ACCESS

Edited by:

Yuji Morita,

Meiji Pharmaceutical University, Japan

Reviewed by:

Marina T. Milenković,

University of Belgrade, Serbia

Dana Marshall,

Meharry Medical College,

United States

*Correspondence:

Renkang Dong

627361728@qq.com

Yu Zhen Shi

418391767@qq.com

Xiu Nian Hu

huxiunian@163.com

Specialty section:

This article was submitted to Infectious Diseases,

a section of the journal

Frontiers in Microbiology

Received: 15 June 2021 Accepted: 19 August 2021

Published: 05 October 2021

Citation:

Liu H, Cao CY, Qiu FL, Huang HN,

Xie HY, Dong RK, Shi YZ and Hu XN

(2021) Iron-Rich Conditions Induce

OmpA and Virulence Changes of Acinetobacter baumannii.

Front. Microbiol. 12:725194.

doi: 10.3389/fmicb.2021.725194

\section{Iron-Rich Conditions Induce OmpA and Virulence Changes of Acinetobacter baumannii}

\author{
Hui Liu', Chun yuan $\mathrm{Cao}^{2}$, Fu lan Qiu', Hao Nan Huang', Hongyan Xie', \\ Renkang Dong ${ }^{1 *}$, Yu Zhen Shi ${ }^{3 *}$ and Xiu Nian $\mathrm{Hu}^{4 *}$
}

'Department of Clinical Laboratory, Fujian Longyan First Hospital/Longyan First Affiliated Hospital of Fujian Medical University, Longyan, China, ${ }^{2}$ Department of Clinical Laboratory, Fujian Longyan Center for Disease Control and Prevention, Longyan, China, ${ }^{3}$ Intensive Care Unit, Fujian Longyan First Hospital/Longyan First Affiliated Hospital of Fujian Medical University, Longyan, China, ${ }^{4}$ Orthopaedic Surgery, Fujian Longyan First Hospital/Longyan First Affiliated Hospital of Fujian Medical University, Longyan, China

Background: Iron ions affect the expression of outer membrane protein A (OmpA), a major pathogenic protein in Acinetobacter baumannii.

Objective: To analyze the effect of iron ions on the expression of the OmpA protein of A. baumannii and explore its association with the virulence of OmpA.

Methods: Site-directed mutagenesis was used to construct ompA gene deletion strains and gene repair strains. The OmpA protein expression of $A$. baumannii under culture with different contents of iron ions was detected. The virulence of $A$. baumannii with different OmpA protein expression levels were evaluated in macrophages and mice.

Results: OmpA protein levels of the three strains were enhanced under iron-rich conditions. They were reduced in the presence of the iron-chelating agent $2,2^{\prime}-$ bipyridine. A. baumannii wild type and + ompA had a remarkable toxic effect on RAW246.7 macrophages $(P<0.05)$. In contrast, the $\triangle$ ompA had a significantly reduced toxic effect on RAW246.7 macrophages $(P<0.05)$. The levels of the inflammatory factors IL-1 $\beta, I L-6, I L-8$, and $T N F \alpha$ in the mice spleen were significantly increased in the + ompA strain treatment group compared with the $\triangle$ ompA strain group (all $P<0.05)$. In addition, the levels were higher in the presence of iron ions than in the presence of the chelating agent.

Conclusion: Iron-rich conditions increase the OmpA protein expression of A. baumannii. Strains with high OmpA protein expression were more invasive, which may be a key determinant of $A$. baumannii infection and pathogenicity. Iron control strategies might be used for the management of $A$. baumannii.

Keywords: A. baumannii, iron, OmpA protein, virulence, mice

\section{INTRODUCTION}

Acinetobacter baumannii is a Gram-negative bacterium among the most important opportunistic pathogens for nosocomial infections, with strong acquired resistance and clonal spread capability (Peleg et al., 2008; Howard et al., 2012; Lee et al., 2017). Due to factors such as mechanical ventilation, use of broad-spectrum antibiotics, length of stay in ICU, and coma, critically ill 
patients with multidrug-resistant and pan drug-resistant A. baumannii infections often have a high mortality rate of 26.0$55.7 \%$, with attributable mortality rates of $8.4-36.5 \%$ (Inchai et al., 2015; Uwingabiye et al., 2016; Xiao et al., 2017). According to the World Health Organization (WHO) report in 2017, carbapenem-resistant $A$. baumannii (CRAB) is currently the bacterium that poses the greatest threat to human health and for which new antibiotics are desperately needed (Willyard, 2017).

The outer membrane (OM) of Gram-negative bacteria is a unique architecture that acts as a defensive barrier to toxic molecules. It is composed of phospholipids, lipopolysaccharide (LPS), outer membrane $\beta$-barrel proteins (OMP), and lipoproteins (Dhilon and Patel, 2017; Choi and Lee, 2019). The outer membrane protein comprises heat-modifying protein, porin, and lipoprotein. They play an essential role in maintaining the bacterial structure, substance transport, cell surface recognition, signal transduction, and pathogenicity. However, they are also involved in physiological functions such as bacterial infection, adhesion, inflammation, activating the host to produce immune protection, and involvement in drug resistance (Nie et al., 2020; Viale and Evans, 2020). The outer membrane protein A (OmpA) is one of the components of OMPs of several Gram-negative bacilli. It is a key virulence factor that mediates bacterial biofilm formation, eukaryotic cell infection, antibiotic resistance, and immune regulation (Lee et al., 2017; Nie et al., 2020; Viale and Evans, 2020). An increase in the secretion of OmpA is independently associated with mortality in pneumonia and bacteremia caused by A. baumannii (SanchezEncinales et al., 2017). In addition, the mRNA expression level of ompA in A. baumannii is considered a rapid diagnostic index for antibiotics resistance (Martin-Pena et al., 2013). The OmpA is essential for A. baumannii to adhere to and invade epithelial cells and disseminate in blood and tissues (Gaddy et al., 2009; Parra-Millan et al., 2018). Therefore, OmpA is an attractive treatment target to fight $A$. baumannii infections (Nie et al., 2020).

Differential proteomics experiments revealed that the iron ion concentration affects the expression of the OmpA protein in A. baumannii (Nwugo et al., 2011). As an essential element and limited micronutrient for the survival of living organisms, iron ions are essential to maintain the activity of many intracellular enzymes (Abbaspour et al., 2014). In an aerobic environment, the iron available to cells is quite limited. After pathogenic bacterial infection, microorganisms must compete with the host to obtain iron (Zughaier and Cornelis, 2018). Mammals can restrict the utilization of iron by pathogenic bacteria through isolating intracellular iron and extracellular chelating iron using glycoproteins, transferrin, and lactoferrin. In contrast, bacteria have evolved a complex iron uptake system for competition with infected hosts (Dorsey et al., 2004).

Thus, traditional treatment strategies cannot effectively treat complicated infections caused by A. baumannii, especially CRAB and OmpA. As one of the most abundant protein in the OMPs of A. baumannii (Uppalapati et al., 2020), it is extensively involved in regulating physiological functions and deserves further research. Therefore, the present study aimed to analyze the effect of iron ions on the expression of the OmpA protein of
A. baumannii and explore its association with the virulence of OmpA in mouse models.

\section{MATERIALS AND METHODS}

\section{Construction of the ompA Gene Deletion Strain $(\triangle o m p A)$ of $A$. baumannii}

The NCBI GenBank was used to retrieve the nucleotide sequence of A. baumannii ompA (GenBank: AY485227.1). Two pairs of specific $\mathrm{PCR}$ primers $(\mathrm{P} 1 \mathrm{~A} / \mathrm{P} 1 \mathrm{~B}$ and $\mathrm{P} 2 \mathrm{~A} / \mathrm{P} 2 \mathrm{~B})$ were designed: ompA-P1A, 5'-GGAAAGTCTATCAAGTGTTTGTAGGATC CAAA-3' $(B a m \mathrm{HI}) ;$ ompA-P1B, 5'-AACTTCTACTACAGGAG CAGCAGGCTCTCGAGAAA-3' (XhoI); ompA-P2A, 5'-ATCA AGCCGTACGTATTATTAGGTGCTCGAGAAA-3' (XhoI); and ompA-P2B, 5' -TTACTGTTCAAACT-3' (XhoI). Using P1A/P1B and $\mathrm{P} 2 \mathrm{~A} / \mathrm{P} 2 \mathrm{~B}$ as primers and the $A$. baumannii standard strain ATCC17978 [American Type Culture Collection (ATCC), United States]as a template, the left and right fragments of the ompA gene were amplified as the homology arms of homologous recombination. The pMD18-T (TaKaRa Bio, Japan) vector and the target fragment were ligated with DNA ligase (TaKaRa Bio, Japan) to transform DH5 $\alpha$ (TaKaRa Bio, Japan) competent cells. Then, the positive plasmids were named p18T-ompA-P1 and p18T-ompA-P2 and verified by sequencing.

The recombinant plasmids p18T-ompA-P1 and p18T-ompAP2 were extracted. BamHI and XhoI was used for double digestion for the p18T-ompA-P1 recombinant plasmid. In contrast, XhoI and SphI was used for double digestion for the p18T-ompA-P2 recombinant plasmid. The pMD18-T vector plasmid was subjected to double digestion with the BamHI and $S p h \mathrm{I}$. The target fragments were purified by a gel extraction kit.

Double digestion at $37^{\circ} \mathrm{C}$ for $3 \mathrm{~h}$ with SphI and BamHI was performed for the $18 \mathrm{~T}$-ompA recombinant plasmid and pDS132 (ATCC, United States) plasmid. The 6,000-bp band of pDS132 double digestion product and the 1,500-bp band of p18TompA double digestion product were extracted. The products extracted from the above two gel extraction kits were ligated overnight at $16^{\circ} \mathrm{C}$. The ligation products were transformed into $E$. coli competent cells and the required pDS132-ompA recombinant plasmid were screened using LB (Sangon Biotech, China) plates containing Amp $+(100 \mu \mathrm{g} / \mathrm{mL})$. Then, a single colony was selected into the LB liquid medium containing the same concentration of Amp + and underwent shaking culture at $37^{\circ} \mathrm{C}$. This was until the optical density (OD) value was about 1.0 , which was measured at $600 \mathrm{~nm}$. The positive recombinant plasmid after PCR and digestion identification was named pDS13-ompA.

For the screening of the $\triangle o m p A$ of $A$. baumannii, it was constructed by conjugative transduction of a double-parent filter membrane (Griffiths et al., 2000). E. coli (pDS132ompA) was used as the donor strain. The A. baumannii standard strain ATCC17978 was used as the recipient strain for conjugative transduction. The identification of the $\Delta o m p A$ strain of $A$. baumannii was performed through a successful growth in Kanamycin $(0.02 \mathrm{mg} / \mathrm{mL})$ resistance selective medium and confirmed using western blot analysis and PCR identification. 


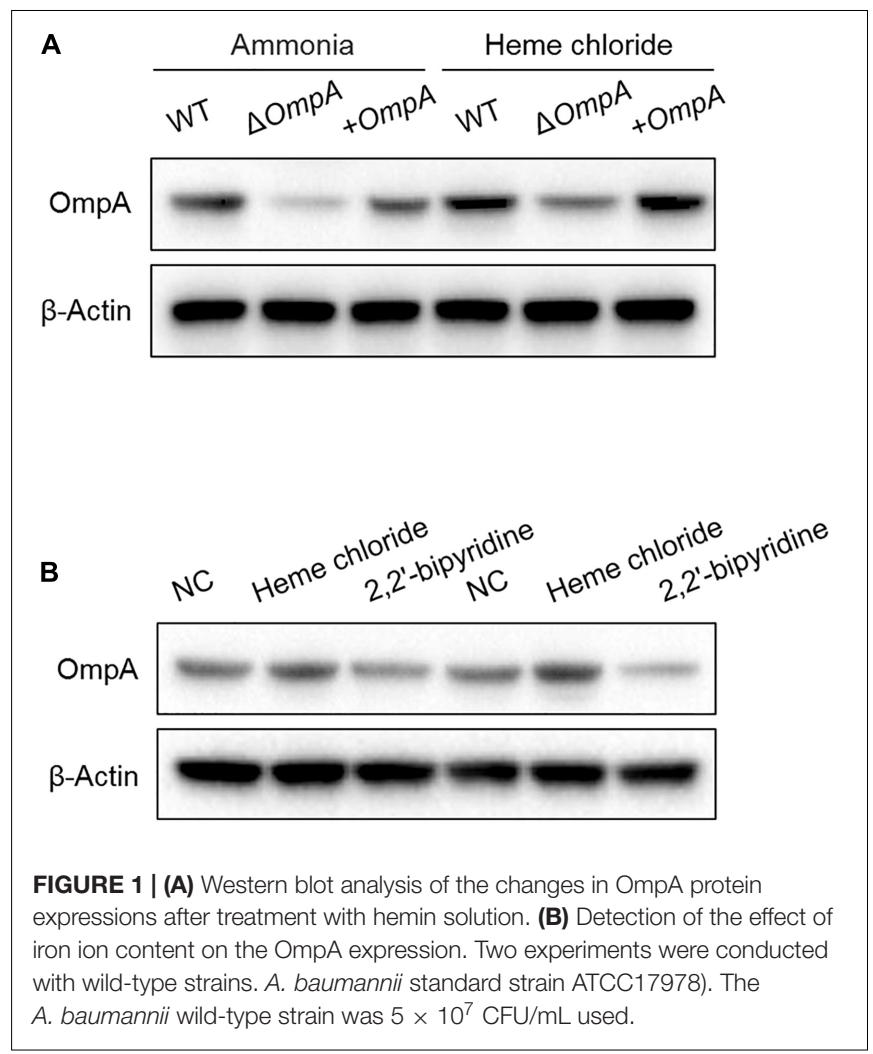

\section{Construction of the ompA Gene Complementation Strain (+ompA) of \\ A. baumannii OmpA}

The primer sequences of the full-length ompA gene were designed and amplified (ompA-F1, 5'-GGAAAGTCTATCAA GTGTTTGTATG-3'; and ompA-R1: 5'-CGAGTCGCTTTTT TACTGTTCA- $3^{\prime}$ ). Using the $A$. baumannii standard strain ATCC17978 as a template, the fragment size was about 1,235 bp. The PCR product of the amplified full-length ompA gene was subjected to gel electrophoresis. The DNA fragment was extracted and ligated to the pMD18-T vector and transformed into $\mathrm{DH} 5 \alpha$ competent cells. The positive clone pT-ompA was selected and submitted to Genewiz Suzhou Biotechnology for sequencing. Double digestion at $37^{\circ} \mathrm{C}$ for $3 \mathrm{~h}$ with $\mathrm{AccI}$ and $B g l I I$ was performed for the $\mathrm{pT}$-ompA recombinant plasmid and pWH1266 (ATCC, United States) vector plasmid.

The 7,000-bp band of the pWH1266 double digestion product and the 1,200-bp band of the pT-ompA double digestion product were extracted. The products extracted from the above two gel extraction kits were ligated overnight at $16^{\circ} \mathrm{C}$, and the ligation products were transformed into $\triangle o m p A$ competent cells. An LB plate containing Amp $+(100 \mu \mathrm{g} / \mathrm{mL})$ was used to screen the required pWH1266-ompA recombinant plasmid. A single colony was selected into the LB liquid medium containing the same concentration of Amp + and underwent shaking culture at $37^{\circ} \mathrm{C}$ until the $\mathrm{OD}$ value was about 1.0 . After western blot and PCR confirmation, the $\triangle o m p A+\mathrm{pWH} 1266-o m p A$ strain $(+o m p A)$ was obtained.

\section{Detection of Effects of Different Contents of Iron Ions on ompA Expression}

The A. baumannii wild-type strain (A. baumannii standard strain ATCC17978), $\triangle o m p A$ strain, and +ompA strain after activation overnight were transferred to the LB culture medium with $2 \mathrm{mM} \mathrm{FeSO}_{4}$ added at 1:100. Then, transferred to the LB culture medium added with $350 \mu \mathrm{M}$ (350 $\mu \mathrm{mol} / \mathrm{L}) 2,2^{\prime}$ bipyridine solution for culture in the shaker at $37^{\circ} \mathrm{C}$ for $8-9 \mathrm{~h}$ and 3-4 h, respectively; then, the bacteria were collected. Western blot was used to detect the OmpA contents in the three groups.

\section{Dimethylthiazol-Diphenyltetrazolium Bromide (MTT) Colorimetry for Cytotoxicity Test}

The concentration of RAW246.7 [National Collection of Authenticated Cell Cultures (NCTC), United Kingdom]macrophages was adjusted to $2 \times 10^{3} / \mathrm{mL}$ with complete culture medium [10\% fetal bovine serum (FBS) and $2 \%$ double-antibody were added to Dulbecco's modified eagle medium (DMEM) purchased from Thermo Fisher Scientific, United States] and seeded in 96-well plates with 100 $\mu \mathrm{L}$ per well. The RAW246.7 macrophages were added with $8 \log \mathrm{CFU} / \mathrm{mL}$ of each $A$. baumannii strain. Normal saline was used as the control group. After $24 \mathrm{~h}$ of treatment, an MTT kit (cell proliferation kit I, Sigma-Aldrich, Germany)was used to detect cell viability. After adding MTT for $2 \mathrm{~h}$, the Vario Skan Flash multi-functional microplate reader (Thermo Fisher Scientific, United States) was used to measure the optical density (OD) value of each well at $450 \mathrm{~nm}$.

\section{Pathogenicity Test in Mice}

Female nude mice (4-6-week-old) were obtained from Vital River Laboratory Animal Technology (Beijing, China) and kept in a specific pathogen-free environment at $25^{\circ} \mathrm{C}, 55 \%$ humidity, and 12-h light cycles with free access sterilized food and water. The experiments were approved by the animal ethics committee of the Ethics Committee of Longyan First Affiliated Hospital of Fujian Medical University, Longyan, Fujian, China (2017026 and 2020067). The mice were checked for their health status, and animal welfare supervision was provided by a certified veterinarian. All animal experiments were carried out in accordance with the Chinese governing laws on the use of medical laboratory animals (authorization no. 551998, 2013, by the Ministry of Health). Two A. baumannii wild-type strains, $\triangle o m p A$ strain, and +ompA strain, were inoculated into LB culture medium. They were placed in a $37^{\circ} \mathrm{C}$ incubator for 20 $24 \mathrm{~h}$ and then mixed with $10 \%(\mathrm{w} / \mathrm{v})$ porcine mucoprotein at 1:1 to prepare a bacterial suspension. The bacterial content was determined by the spread plate method. The minimum lethal concentration and the maximum non-lethal concentration of the isolates for mice were first measured through preliminary testing. Then formal testing was carried out on this basis. First, 20 mice were randomly assigned to five groups (the experimental groups) and then administrated with $1 \mathrm{~mL}$ of normal saline and $1 \mathrm{~mL}$ of a bacterial suspension at an intraperitoneal injection dose of 

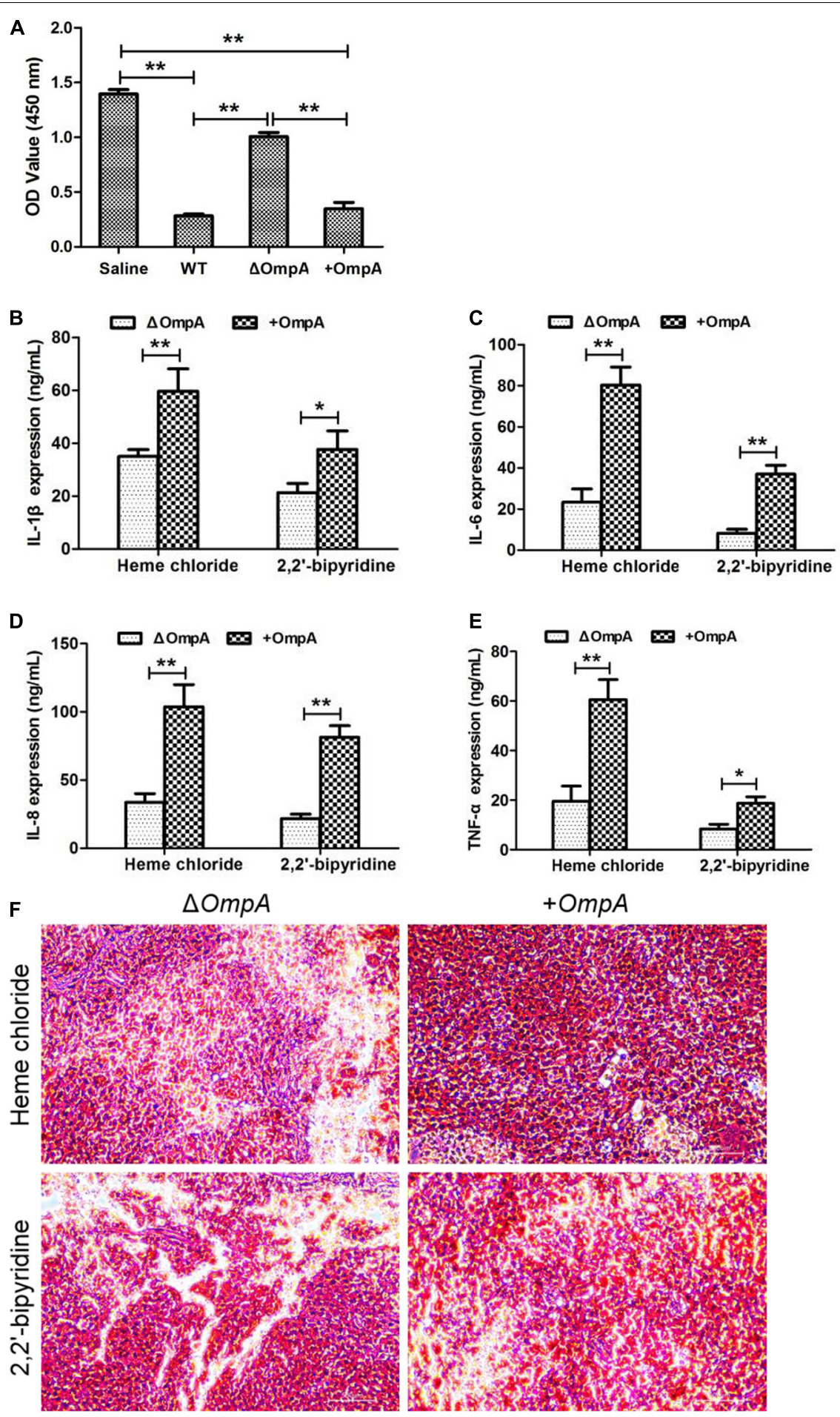

FIGURE 2 | (A) Toxic effect of the $\triangle O m p A$ and + ompA strains on macrophages. (B-E) The expression levels of inflammatory factors IL-1 $\beta$, IL-6, IL-8, and TNF $\alpha$ in the mice spleen were significantly increased in the $+\mathrm{O} p \mathrm{~A}$ strain treatment group compared with the $\triangle O m p A$ strain group. (F) The picture of spleen tissue grinding and Gram staining. Cells stained in red are bacteria. ${ }^{*} P<0.05$ and ${ }^{\star \star} P<0.01$.

$5.7 \times 10^{8} \mathrm{CFU} / \mathrm{mL}, 1.14 \times 10^{8} \mathrm{CFU} / \mathrm{mL}, 2.28 \times 10^{7} \mathrm{CFU} / \mathrm{mL}$, $4.56 \times 10^{6} \mathrm{CFU} / \mathrm{mL}$, and $9.12 \times 10^{5} \mathrm{CFU} / \mathrm{mL}$. The blank control group was injected with $1 \mathrm{~mL}$ of normal saline. After 7 days of observation, the disease onset and death of mice and pathological necropsy changes were recorded. The modified Karber method (Rath et al., 2011) was used to calculate the LD50. The mice 
TABLE 1 | The pathogenicity of $A$. baumannii wild, $\triangle o m p A$, and +ompA strain in mice.

\begin{tabular}{|c|c|c|c|c|c|c|c|c|}
\hline \multirow[t]{2}{*}{ Group } & \multirow[t]{2}{*}{ Dose/(CFU/mL) } & \multirow[t]{2}{*}{$\begin{array}{l}\text { No. of animals } \\
\text { in each strain }\end{array}$} & \multicolumn{2}{|c|}{$\begin{array}{l}\text { Deaths and the } \\
\text { mortality rate of } \\
\text { A. baumannii wild }\end{array}$} & \multicolumn{2}{|c|}{$\begin{array}{l}\text { Deaths and the } \\
\text { mortality rate of } \Delta \\
\text { ompA }\end{array}$} & \multicolumn{2}{|c|}{$\begin{array}{l}\text { Deaths and the } \\
\text { mortality rate } \\
\text { of }+ \text { ompA }\end{array}$} \\
\hline & & & Deaths & Mortality rate & Deaths & Mortality rate & Deaths & Mortality rate \\
\hline 1 & $5 \times 10^{8}$ & 4 & 4 & $100 \%$ & 1 & $25 \%$ & 3 & $75 \%$ \\
\hline 2 & $1 \times 10^{8}$ & 4 & 4 & $100 \%$ & 1 & $25 \%$ & 2 & $50 \%$ \\
\hline 3 & $5 \times 10^{7}$ & 4 & 2 & $50 \%$ & 0 & $0 \%$ & 2 & $50 \%$ \\
\hline 4 & $1 \times 10^{6}$ & 4 & 1 & $25 \%$ & 0 & 0 & 1 & $25 \%$ \\
\hline 5 & $5 \times 10^{5}$ & 4 & 0 & 0 & 0 & 0 & 0 & 0 \\
\hline 8 & Normal saline & 4 & 0 & 0 & 0 & 0 & 0 & 0 \\
\hline
\end{tabular}

CFU, colony-forming unit.

were sacrificed by cervical dislocation, and the spleens were immediately harvested and ground. Smears were prepared and stained with the Gram solution to observe the bacteria.

Spleen tissue homogenates $(10 \%, \mathrm{wt} / \mathrm{vol})$ were prepared with cold $1 \times$ PBS ( $\mathrm{pH}$ 7.2). TNF- $\alpha$, IL-1 $\beta$, IL-6, and IL-8 levels were measured in spleen tissues using mice enzyme-linked immunosorbent assay kits (Thermo Fisher Scientific, Waltham, MA, United States) according to the instructions.

\section{Western Blot Analysis}

The strain of A. baumannii was lysed and collected with Bacterial Lysis Buffer (Cat\# C500003, Sangon Biotech, China) supplemented with protease inhibitor cocktail (Cat\# P8340, Roche). The lysis products were electrophoresed by sodium dodecyl sulfate-polyacrylamide gel electrophoresis (SDSPAGE) and then transferred to polyvinylidene fluoride (PVDF) membranes. Incubation of the suggested primary antibodies OmpA (Abcam) and secondary antibodies (Abcam) was implemented in a $5 \%$ milk blocking reagent. Autoradiograms were quantified through densitometry controlled by GAPDH. The assay was repeated three times.

\section{qRT-PCR}

Complete RNA was extracted from the strain of A. baumannii with TRIzol reagent (Cat\# 15596018, Life Technologies) in keeping with the manufacturer's instructions. Total RNA of 2 $\mu \mathrm{g}$ was reverse transcribed according to the protocol for the PrimeScript $^{\circledR}$ RT Master Mix Perfect Real-Time (Cat\# RR047A, TaKaRa Bio, Japan). The qPCR reactions were implemented with SYBR Green Master Mix (Cat\# RR820A, TaKaRa Bio, Japan) on a quantitative PCR instrument (Applied Biosystems 7900HT, Thermo Fisher Scientific).

\section{Statistical Analysis}

All statistical analyses were performed using SPSS 22.0 (IBM, Armonk, NY, United States) and GraphPad Prism 5 (GraphPad Software Inc., San Diego, CA, United States). All continuous data are presented as means \pm standard deviations and were analyzed using Student's $t$-test or ANOVA with the LSD post hoc test. $P$-values $<0.05$ were considered statistically significant.

\section{RESULTS}

\section{Detection of Effects of Iron Ion Contents on OmpA Expression}

The A. baumannii wild-type strain was $5 \times 10^{7} \mathrm{CFU} / \mathrm{mL}$ used. The ompA expression of $A$. baumannii cultured in the LB culture medium added with $2 \mathrm{mM} \mathrm{FeSO}_{4}$ was higher than that cultured after addition with $350 \mu \mathrm{M}(350 \mu \mathrm{mol} / \mathrm{L})$ of the iron-chelating agent 2,2' -bipyridine (Figure 1).

\section{Toxic Effect of $\triangle$ ompA Strain and + ompA Strain on Macrophages}

The results of the MTT test showed that the A. baumannii wild type and $+o m p A$ bacterial suspensions had a remarkable toxic effect on the RAW246.7 macrophages $(P<0.05)$. On the other hand, the $\triangle o m p A$ bacterial suspension had a significantly reduced toxic effect on the RAW246.7 macrophages $(P<0.05)$ (Figure 2A).

\section{Toxic Effect of $\triangle$ ompA Strain and +ompA Strain in Mice}

The LD50 of the A. baumannii wild-type strain was $5 \times 10^{7} \mathrm{CFU} / \mathrm{mL}$ (Table 1 ). The LD50 of the $\triangle o m p A$ strain was $>5 \times 10^{8} \mathrm{CFU} / \mathrm{mL}$ (Table 1). The LD50 of the + ompA strain was $5 \times 10^{7}-1 \times 10^{8} \mathrm{CFU} / \mathrm{mL}$ (Table 1 ).

The levels of the inflammatory factors IL-1 $\beta$, IL-6, IL-8, and $\mathrm{TNF} \alpha$ and the number of viable bacteria in the mice spleen were significantly increased in the $+o m p A$ strain treatment group compared with the $\triangle o m p A$ strain group (all $P<0.05$ ) (Figures 2B-F). In addition, the levels were higher in the presence of iron ions than in the presence of the chelating agent. The invasion of the spleen by the $+o m p A$ strain was higher than by the $\triangle o m p A$ strain and higher in the presence of iron than in the presence of the chelating agent. The details of strain construction are provided in Supplementary Data and Supplementary Figure 1.

\section{DISCUSSION}

A. baumannii plays an important role in nosocomial infections (Peleg et al., 2008; Howard et al., 2012; Lee et al., 2017; 
Wong et al., 2017; Harding et al., 2018). Iron ions affect the expression of OmpA, which is a major pathogenic protein in A. baumannii. This study aimed to analyze the effect of iron ions on the expression of the OmpA protein of A. baumannii and explore its association with the virulence of OmpA. The results suggest that iron-rich conditions increase the OmpA protein expression of A. baumannii. Strains with high OmpA protein expression were more invasive, which may be a key determinant of A. baumannii infection and pathogenicity. Iron control strategies might be used for the management of $A$. baumannii.

The OmpA is one of the most abundant protein in the outer membrane of $A$. baumannii. Its molecular weight is $38 \mathrm{kD}$ (Maiti et al., 2011). The $\mathrm{N}$-terminal domain is a $\beta$ barrel structure formed by 8 -strand anti-parallel folding. The C-terminus is embedded in the peptidoglycan layer of the bacterial membrane and participates in the transmembrane transport of hydrophobic molecules (Jahangiri et al., 2017). AbOmpA amino acids isolated from various clinical strains are highly conserved (similarities $>89 \%$ ), but they are heterologous for the human proteome (Jahangiri et al., 2017). When invasive infection occurs, A. baumannii first attaches to the host cell and then invades and transfers to the nucleus. After killing the host cell, it spreads in the bloodstream and tissues (Parra-Millan et al., 2018). In this process, OmpA is involved in mediating the adhesion and invasion of A. baumannii to epithelial cells (Schweppe et al., 2015). At the same time, OmpA contains abundant antigenic sites, which can induce host immunity in multiple ways and play an important role in A. baumannii control after colonization (McConnell et al., 2013).In order to further understand the function of OmpA, the present study analyzed the response of the A. baumannii ompA gene after inactivation and repair to host infection and pathogenicity. The present study showed that ompA deletion decreased the infectivity of A. baumanniiin vitro and in vivo. At the same time, repair increased infectivity, as supported by various studies (MartinPena et al., 2013; Lee et al., 2017; Sanchez-Encinales et al., 2017; Viale and Evans, 2020).

Still, this infectivity results from many complex factors, including iron (Gaddy et al., 2009), which is tightly controlled by hosts for aerobic energy production and to control infections (Abbaspour et al., 2014). Still, bacteria have evolved a complex iron uptake system to compete with the infected host (Dorsey et al., 2004). The free iron concentration in bacterial cells is mainly regulated by the iron uptake regulator (Fur). When the free iron concentration in the cell elevates, the Fur gene can bind to ferrous ions. Thereby inhibiting the genes coding of the iron uptake system and stimulating the genes coding the iron storage protein (Fontenot et al., 2020). In humans, transferrin prevents pathogens from contacting iron ions (Khan et al., 2018). The present study transferred various strains to LB culture medium added with $2 \mathrm{mM} \mathrm{FeSO}_{4}$. It then depleted free iron content in the cells using the iron-chelating agent 2,2'-bipyridine. Western blot suggested that the synthesis and expression of OmpA in the three groups were enhanced under ironrich conditions. They were reduced in the presence of the iron-chelating agent, which confirms that the down-regulated phenotype of OmpA expression is caused, at least in part, by iron limitation.

In the in vitro pathogenicity study, the virulence test of A. baumannii on the RAW246.7 macrophages showed that the A. baumannii strain with OmpA was more invasive than that without OmpA, which had been further proved in the lethal infection experiment in mice. OmpA is needed for A. baumannii invasion (Gaddy et al., 2009), and mutations in ompA decrease the A. baumannii burden in mice (Choi et al., 2008). AbOmpA can directly cause the death of cells (Jin et al., 2011). AbOmpA also stimulates the innate immune response, as shown by higher inflammatory cytokines in the spleen of the mice (Akira and Hemmi, 2003; Trinchieri and Sher, 2007). In both cases, the virulence was higher in the presence of iron and lowered in the presence of the chelating agent. These results suggest that OmpA is a target for the management of A. baumannii infections and that iron-chelating agents could play a role in infection management, especially in the presence of pan drug-resistant A. baumannii. The actual methods that are being sought include OmpA -binding peptides, vaccines targeting OmpA epitopes, and monoclonal antibodies directly targeting OmpA (Nie et al., 2020). This study further suggests that iron chelation could also be used (Gentile et al., 2014).

This study has limitations. It only analyzed the effects of iron ions on the OmpA protein expression of A. baumannii and the pathogenicity of strains with different OmpA expressions. Whether iron-rich or iron-limited conditions promote the biofilm formation of $A$. baumannii, resistance to oxidative stress and other factors that affect the virulence phenotype remain to be studied.

In conclusion, the present study suggests that ironrich conditions promote the OmpA protein expression of A. baumannii. The strains with high OmpA protein expression are more invasive, which may be a key determinant of A. baumannii infection and pathogenicity. Iron-dependent control strategies might be used to treat infectious diseases caused by pathogenic microorganisms.

\section{DATA AVAILABILITY STATEMENT}

The original contributions presented in the study are included in the article/ Supplementary Material, further inquiries can be directed to the corresponding author/s.

\section{ETHICS STATEMENT}

The animal study was reviewed and approved by the animal ethics committee of the Ethics Committee of Longyan First Affiliated Hospital of Fujian Medical University.

\section{AUTHOR CONTRIBUTIONS}

HL: manuscript preparation and funds collection. CYC: experiment involve. FLQ: literature search. HNH: statistical 
analysis. HYX: data collection. RKD: data interpretation. YZS: data interpretation. XNH: study design and data interpretation. All authors contributed to the article and approved the submitted version.

\section{FUNDING}

This work was supported by the Fujian Province Natural Science Foundation (Grant No. 2018J01408) and the Fujian Provincial Health Technology Project (Grant No. 2016-1-97).

\section{REFERENCES}

Abbaspour, N., Hurrell, R., and Kelishadi, R. (2014). Review on iron and its importance for human health. J. Res. Med. Sci. 19, 164-174.

Akira, S., and Hemmi, H. (2003). Recognition of pathogen-associated molecular patterns by TLR family. Immunol. Lett. 85, 85-95. doi: 10.1016/s0165-2478(02) 00228-6

Choi, C. H., Lee, J. S., Lee, Y. C., Park, T. I., and Lee, J. C. (2008). Acinetobacter baumannii invades epithelial cells and outer membrane protein A mediates interactions with epithelial cells. BMC Microbiol. 8:216. doi: 10.1186/14712180-8-216

Choi, U., and Lee, C-R., (2019). Antimicrobial Agents That Inhibit the Outer Membrane Assembly Machines of Gram-Negative Bacteria. J. Microbiol. Biotechnol. 29, 1-10. doi: 10.4014/jmb.1804. 03051

Dhilon, S., and Patel, Y. I. (2017). Wonpil Im. Modeling and simulation of bacterial outer membranes and interactions with membrane proteins. Curr. Opin. Struct. Biol. 43, 131-140. doi: 10.1016/j.sbi.2017.01.003

Dorsey, C. W., Tomaras, A. P., Connerly, P. L., Tolmasky, M. E., Crosa, J. H., and Actis, L. A. (2004). The siderophore-mediated iron acquisition systems of Acinetobacter baumannii ATCC 19606 and Vibrio anguillarum 775 are structurally and functionally related. Microbiology 150, 3657-3667. doi: 10 . 1099/mic.0.27371-0

Fontenot, C. R., Tasnim, H., Valdes, K. A., Popescu, C. V., and Ding, H. (2020). Ferric uptake regulator (Fur) reversibly binds a [2Fe-2S] cluster to sense intracellular iron homeostasis in Escherichia coli. J. Biol. Chem. 295, 1545415463. doi: 10.1074/jbc.ra120.014814

Gaddy, J. A., Tomaras, A. P., and Actis, L. A. (2009). The Acinetobacter baumannii 19606 OmpA protein plays a role in biofilm formation on abiotic surfaces and in the interaction of this pathogen with eukaryotic cells. Infect. Immun. 77, 3150-3160. doi: 10.1128/iai.00096-09

Gentile, V., Frangipani, E., Bonchi, C., Minandri, F., Runci, F., and Visca, P. (2014). Iron and Acinetobacter baumannii Biofilm Formation. Pathogens 3, 704-719. doi: 10.3390/pathogens3030704

Griffiths, A. J. F., Miller, J. H., and Suzuki, D. T. (2000). An Introduction to Genetic Analysis, 7th Edn. New York: W. H. Freeman.

Harding, C. M., Hennon, S. W., and Feldman, M. F. (2018). Uncovering the mechanisms of Acinetobacter baumannii virulence. Nat. Rev. Microbiol. 16, 91-102. doi: 10.1038/nrmicro.2017.148

Howard, A., O’Donoghue, M., Feeney, A., and Sleator, R. D. (2012). Acinetobacter baumannii: an emerging opportunistic pathogen. Virulence 3, 243-250. doi: 10.4161/viru. 19700

Inchai, J., Pothirat, C., Bumroongkit, C., Limsukon, A., Khositsakulchai, W., and Liwsrisakun, C. (2015). Prognostic factors associated with mortality of drugresistant Acinetobacter baumannii ventilator-associated pneumonia. J. Intensive Care 3:9. doi: 10.1186/s40560-015-0077-4

Jahangiri, A., Rasooli, I., Owlia, P., Fooladi, A. A., and Salimian, J. (2017). In silico design of an immunogen against Acinetobacter baumannii based on a novel model for native structure of Outer membrane protein A. Microb. Pathog. 105, 201-210. doi: 10.1016/j.micpath.2017.02.028

Jin, J. S., Kwon, S. O., Moon, D. C., Gurung, M., Lee, J. H., Kim, S. I., et al. (2011). Acinetobacter baumannii secretes cytotoxic outer membrane protein A

\section{ACKNOWLEDGMENTS}

We would like to thank all participants of the study for their hard work.

\section{SUPPLEMENTARY MATERIAL}

The Supplementary Material for this article can be found online at: https://www.frontiersin.org/articles/10.3389/fmicb. 2021.725194/full\#supplementary-material

via outer membrane vesicles. PLoS One 6:e17027. doi: 10.1371/journal.pone. 0017027

Khan, A., Singh, P., and Srivastava, A. (2018). Synthesis, nature and utility of universal iron chelator - Siderophore: A review. Microbiol. Res. 21, 103-111. doi: 10.1016/j.micres.2017.10.012

Lee, C. R., Lee, J. H., Park, M., Park, K. S., Bae, I. K., Kim, Y. B., et al. (2017). Biology of Acinetobacter baumannii: Pathogenesis, Antibiotic Resistance Mechanisms, and Prospective Treatment Options. Front. Cell Infect. Microbiol. 7:55.

Maiti, B., Shetty, M., Shekar, M., and Karunasagar, I. (2011). Recombinant outer membrane protein A (OmpA) of Edwardsiella tarda, a potential vaccine candidate for fish, common carp. Microbiol. Res. 167, 1-7. doi: 10.1016/j.micres. 2011.02.002

Martin-Pena, R., Dominguez-Herrera, J., Pachon, J., and McConnell, M. J. (2013). Rapid detection of antibiotic resistance in Acinetobacter baumannii using quantitative real-time PCR. J. Antimicrob. Chemother. 68, 1572-1575. doi: 10.1093/jac/dkt057

McConnell, M. J., Actis, L., and Pachon, J. (2013). Acinetobacter baumannii: human infections, factors contributing to pathogenesis and animal models. FEMS Microbiol. Rev. 37, 130-155. doi: 10.1111/j.1574-6976.2012.00344.x

Nie, D., Hu, Y., Chen, Z., Li, M., Hou, Z., Luo, X., et al. (2020). Outer membrane protein A (ompA) as a potential therapeutic target for Acinetobacter baumannii infection. J. Biomed. Sci. 27:26.

Nwugo, C. C., Gaddy, J. A., Zimbler, D. L., and Actis, L. A. (2011). Deciphering the iron response in Acinetobacter baumannii: A proteomics approach. J. Proteomics 74, 44-58. doi: 10.1016/j.jprot.2010.07.010

Parra-Millan, R., Guerrero-Gomez, D., Ayerbe-Algaba, R., Pachon-Ibanez, M. E., Miranda-Vizuete, A., Pachon, J., et al. (2018). Intracellular Trafficking and Persistence of Acinetobacter baumannii Requires Transcription Factor EB. mSphere 2018:3.

Peleg, A. Y., Seifert, H., and Paterson, D. L. (2008). Acinetobacter baumannii: emergence of a successful pathogen. Clin. Microbiol. Rev. 21, 538-582. doi: $10.1128 / \mathrm{cmr} .00058-07$

Rath, S., Sahu, M. C., Dubey, D., Debata, N. K., and Padhy, R. N. (2011). Which value should be used as the lethal concentration 50 (LC(50)) with bacteria? Interdiscip. Sci. 3, 138-143. doi: 10.1007/s12539-011-0081-x

Sanchez-Encinales, V., Alvarez-Marin, R., Pachon-Ibanez, M. E., FernandezCuenca, F., Pascual, A., Garnacho-Montero, J., et al. (2017). Overproduction of Outer Membrane Protein A by Acinetobacter baumannii as a Risk Factor for Nosocomial Pneumonia, Bacteremia, and Mortality Rate Increase. J. Infect. Dis. 215, 966-974.

Schweppe, D. K., Harding, C., Chavez, J. D., Wu, X., Ramage, E., Singh, P. K., et al. (2015). Host-Microbe Protein Interactions during Bacterial Infection. Chem. Biol. 22, 1521-1530. doi: 10.1016/j.chembiol.2015.09.015

Trinchieri, G., and Sher, A. (2007). Cooperation of Toll-like receptor signals in innate immune defence. Nat. Rev. Immunol. 7, 179-190. doi: 10.1038/nri2038

Uppalapati, S. R., Sett, A., and Pathania, R. (2020). The Outer Membrane Proteins OmpA, CarO, and OprD of Acinetobacter baumannii Confer a Two-Pronged Defense in Facilitating Its Success as a Potent Human Pathogen. Front. Microbiol. 11:589234.

Uwingabiye, J., Frikh, M., Lemnouer, A., Bssaibis, F., Belefquih, B., Maleb, A., et al. (2016). Acinetobacter infections prevalence and frequency of the antibiotics 
resistance: compArative study of intensive care units versus other hospital units. Pan. Afr. Med. J. 23:191.

Viale, A. M., and Evans, B. A. (2020). Microevolution in the major outer membrane protein OmpA of Acinetobacter baumannii. Microb. Genom. 2020:6.

Willyard, C. (2017). The drug-resistant bacteria that pose the greatest health threats. Nature 543:15. doi: 10.1038/nature.2017.21550

Wong, D., Nielsen, T. B., Bonomo, R. A., Pantapalangkoor, P., Luna, B., and Spellberg, B. (2017). Clinical and Pathophysiological Overview of Acinetobacter Infections: a Century of Challenges. Clin. Microbiol. Rev. 30, 409-447. doi: $10.1128 / \mathrm{cmr} .00058-16$

Xiao, D., Wang, L., Zhang, D., Xiang, D., Liu, Q., and Xing, X. (2017). Prognosis of patients with Acinetobacter baumannii infection in the intensive care unit: A retrospective analysis. Exp. Ther. Med. 13, 1630-1633. doi: 10.3892/etm.2017. 4137

Zughaier, S. M., and Cornelis, P. (2018). Editorial: Role of Iron in Bacterial Pathogenesis. Front. Cell Infect. Microbiol. 8:344.
Conflict of Interest: The authors declare that the research was conducted in the absence of any commercial or financial relationships that could be construed as a potential conflict of interest.

Publisher's Note: All claims expressed in this article are solely those of the authors and do not necessarily represent those of their affiliated organizations, or those of the publisher, the editors and the reviewers. Any product that may be evaluated in this article, or claim that may be made by its manufacturer, is not guaranteed or endorsed by the publisher.

Copyright (C) 2021 Liu, Cao, Qiu, Huang, Xie, Dong, Shi and Hu. This is an openaccess article distributed under the terms of the Creative Commons Attribution License (CC BY). The use, distribution or reproduction in other forums is permitted, provided the original author(s) and the copyright owner(s) are credited and that the original publication in this journal is cited, in accordance with accepted academic practice. No use, distribution or reproduction is permitted which does not comply with these terms. 